\title{
Global downregulation of pigmentation-associated genes in human premature hair graying
}

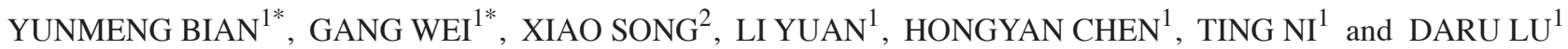 \\ ${ }^{1}$ State Key Laboratory of Genetic Engineering and Ministry of Education Key Laboratory of Contemporary Anthropology, \\ Institute of Genetics, School of Life Sciences, Fudan University, Shanghai 200438; ${ }^{2}$ Department of Thoracic Surgery, \\ Shanghai Pulmonary Hospital, Tongji University, Shanghai 200433, P.R. China
}

Received May 21, 2018; Accepted September 13, 2018

DOI: $10.3892 /$ etm.2019.7663

\begin{abstract}
Premature hair graying, or canities, is a complex multi-factorial process with negative effects on affected individuals. The aim of the present study was to investigate the possible underlying mechanisms of premature hair graying at the genetic level. A total of 5 unrelated Han Chinese individuals presenting with premature hair graying (25-40 years old, with $>1 \%$ hair affected) were enrolled in the present study. RNA sequencing was performed to identify gene expression changes between the follicular cells of grey and black hair from the cohort. A total of 127 differentially expressed genes (DEGs) were identified. These DEGs were overrepresented in categories associated with the pigmentation pathway, with a decreased expression of key genes responsible for melanin synthesis. Of note, the decreased expression of certain transcription factors and the increased expression of certain precursor microRNAs observed may explain for the downregulation of certain other DEGs, which were identified as their targets via Starbase v2 and Integrated Motif Activity Response Analysis. The DEGs were also enriched in terms associated with the nervous system, indicating that neural disturbances may also have certain roles in premature hair graying. Of note, five of the downregulated DEGs were associated with aging according to the JenAge Aging Factor Database. To the best of our knowledge, the present study was the first genome-wide survey of the gene expression profile associated with premature hair graying. Dysfunction of the melanin biosynthesis pathway is probably the direct cause of hair graying and the
\end{abstract}

Correspondence to: Professor Ting Ni or Professor Daru Lu, State Key Laboratory of Genetic Engineering and Ministry of Education Key Laboratory of Contemporary Anthropology, Institute of Genetics, School of Life Sciences, Fudan University, 2005 Songhu Road, Shanghai 200438, P.R. China

E-mail: tingni@fudan.edu.cn

E-mail:drlu@fudan.edu.cn

*Contributed equally

Key words: premature hair graying, gene expression changes, melanogenesis pathway, microRNA, nervous system present results provide valuable clues for further functional and mechanistic investigation.

\section{Introduction}

Hair graying may be classified as natural senile canities and premature graying. Natural senile canities usually has its onset after the age of 40 years and aggravates with the ongoing aging process (1). Unlike senile canities, premature graying occurs prior to the age of 25 years and is usually progressive and permanent $(2,3)$. Although hair graying is considered to be genetically controlled and inheritable, the underlying mechanisms have remained largely elusive (4).

Hair color is determined by pigment granules in hair follicles, wherein melanin synthesis is particularly crucial (5). Mature melanocytes are densely distributed in hair bulbs to sustain active melanogenesis, which is strictly coupled to the anagen stage of the hair cycle $(6,7)$. The biosynthesis of melanin and its subsequent transfer from melanocyte to hair bulb keratinocytes is a rather complex process (8). Previous molecular study has mainly focused on identifying genes that encode characteristic markers for pigmentation, including tyrosinase (TYR), OCA2 melanosomal transmembrane protein, TYR-related protein 1 (TYRP1) and solute carrier family 45 member 2 (SLC45A2) (9). Polymorphisms within these loci are associated with a normal variation in hair color traits (10). In addition, a genome-wide association study on the genetic basis of pigmentation in human subjects revealed that the single nucleotide polymorphism frequency distribution of these genes is linked to skin color and hair color $(11,12)$. A broader study indicated that mutations in two pore segment channel 2, agouti signaling protein and melanocortin 1 receptor are associated with hair color and pigmentation (13).

Previous studies have focused on identifying genes that encode characteristic markers for premature graying of hair. A germline mutation in the syntaxin 17 gene of horses was recently identified to cause premature graying of hair (14) and telomerase reverse transcriptase mutation carriers may also present with premature hair graying (15). Reverse transcription polymerase chain reaction (RT-PCR) arrays on the gene expression profiles of the hair bulge and hair bulb revealed a significant downregulation of melanogenesis associated genes [tyrosinase (TYR), tyrosine related protein 1 (TYRP1), 
melanocyte inducing transcription factor, paired box gene 3 (PAX3) and proopiomelanocortin] in unpigmented hair bulbs and a downregulation of marker genes typical for melanocyte precursor cells [PAX3, SRY-Box 10 (SOX10) and dopachrome tautomerase) in unpigmented mid-segments compared with their pigmented analogues. Superoxide dismutase 3 transgenic mice exhibited premature aging, including hair graying, abnormal gait and a shortened life span (16).

Although previous studies provided candidate genes associated with hair graying, their results are limited due to the unavailability of related hereditary family members or an insufficient number of subjects to provide representative results (17). According to the central dogma of genetics, the genotype affects the phenotype through gene expression. Thus, it is straightforward to assume that a variance in gene expression between the grey and black hair follicles underlies the difference in hair color. However, to the best of our knowledge, no previous study has assessed premature hair graying from this genetic aspect. In the present study, an RNA sequencing (RNA-seq) analysis was performed to reveal gene expression changes between grey and black hair follicles from Han Chinese patients suffering from premature hair graying. It was intended to unravel the underlying mechanisms and potential candidate genes responsible for hair pigmentation loss.

\section{Materials and methods}

Subjects. A total of 5 unrelated Han Chinese donors who presented with premature graying since they were teenagers and had a clear family history of premature hair graying were enrolled in the present study. Their details are specified in (Table I). A total of 30 grey and 30 black hair follicles were randomly collected from each subject and stored in RNAlater ${ }^{\circledR}$ Stabilization Solution (Thermo Fisher Scientific, Inc., Waltham, MA, USA) at $4^{\circ} \mathrm{C}$.

RNA extraction, library preparation and sequencing. Total RNA was extracted from each sample using the mirVana ${ }^{\mathrm{TM}}$ miRNA Detection Kit (Thermo Fisher Scientific, Inc.) in strict accordance with the manufacturer's protocol. The RNA-seq library was prepared using an Ion Total RNA-seq Kit v2 (Thermo Fisher Scientific, Inc.) following the manufacturer's protocol. RNA-seq libraries were sequenced on an Ion Proton $^{\text {TM }}$ System (Thermo Fisher Scientific, Inc.).

Bioinformatics analysis of RNA-seq data. Raw sequencing reads were aligned to the human genome (GRCh38/hg38) using STAR (Spliced Transcripts Alignment to a Reference software; http://code.google.com/p/rna-star/) (18). Ensembl gene annotation (http://www.ensembl.org/info/genome/genebuild/genome annotation.html; release 79) was used for evaluating gene expression using Cufflinks (19), and differentially expressed genes (DEGs) between grey and black hair follicles were determined by Cuffdiff, a subpackage of Cufflinks. The ratio of Fragments per kilobase per million of grey to black hair was calculated and differential expression genes were then studied by $\log 2 \mathrm{FC}$. The criteria used to define DEGs were as follows: i) Fragments per kilobase per million mapped reads $>1$ in at least one sample of a sample pair; ii) fold change of at least 2 and iii) $\mathrm{P}<0.05$.
Functional classification and enrichment analysis of the DEGs were performed using the online platform of the Database for Annotation, Visualization and Integrated Discovery (20). Gene Ontology (GO) terms were used to classify the DEGs. Association between microRNAs (miRs) and their targets were predicted by Starbase v2 (21). Transcription factors (TFs) and their target genes were predicted through Integrated Motif Activity Response Analysis (22). The Search Tool for the Retrieval of Interacting Genes/Proteins (STRING) database (23) was used to construct protein-protein interaction (PPI) networks the DEGs are involved in.

RT-quantitative (q)PCR. Total RNA was extracted from each hair follicle sample. The RNA $(1 \mu \mathrm{g})$ was reverse-transcribed using a ReverTra Ace qPCR RT Kit (Toyobo, Osaka, Japan). $\beta$-actin was used as reference control. SYBR Green Realtime PCR Master Mix (ROX; Toyobo) was applied for PCR amplifications on a Bio-Rad CFX96 system (Bio-Rad Laboratories, Inc., Hercules, CA, USA) according to the manufacturer's protocol. The thermocycling program was as follows: $95^{\circ} \mathrm{C}$ for $5 \mathrm{~min}$, followed by 40 cycles of $95^{\circ} \mathrm{C}$ for $30 \mathrm{sec}, 60^{\circ} \mathrm{C}$ for $40 \mathrm{sec}$ and $72^{\circ} \mathrm{C}$ for $45 \mathrm{sec}$. The primers used are listed in Table II.

\section{Results}

Summary of RNA-seq data. To identify changes in the gene expression profile associated with premature hair greying, RNA-seq libraries derived from each grey or black hair follicle were sequenced using the Ion Proton ${ }^{\mathrm{TM}}$ System (Thermo Fisher Scientific, Inc.). The number of raw sequenced reads for each sample ranged from 15.9 to 27.9 million, and the mapping percentages were 72.1-80.0\%, which demonstrated an overall good mappability.

Gene expression changes between grey and black hair follicles. In order to identify genes with a role in premature hair greying, the samples were classified into a grey hair group and a black hair group, and the gene expression changes were compared between them. With the criteria of a 2-fold change in expression and $\mathrm{P}<0.05$, a total of 127 DEGs were identified, including 47 upregulated and 80 downregulated DEGs (Fig. 1A). In order to confirm these DEGs, the changes in their expression were also assessed for each subject individually. Most of these DEGs were changed in the same direction among the individual subjects (Fig. 1B), demonstrating a consistence regarding the DEGs identified.

To determine the functional roles of these DEGs, the functional categories they were involved in were then examined. Of note, the DEGs were mainly enriched in functional categories closely linked to pigment synthesis or metabolic pathways (Fig. 1C). The DEGs were also known to be located in pigment granules and melanosomes (Fig. 1D), which provided an explanation for the downregulation of these genes in grey hair follicles that had lost their ability to produce melanin, which lead to hair graying. Of note, genes associated with the nervous system accounted for the largest number of DEGs, although the significance of their enrichment was not as high as that in the categories associated with pigmentation. This may imply a disturbance of the nervous system in grey hair follicles compared with that in black ones. 
Table I. Characteristics of the subjects affected by premature hair greying.

\begin{tabular}{lccl}
\hline Gender & Age (years) & Onset age (years) & Head areas affected \\
\hline Male & 31 & 15 & Parietal, frontal and temporal region \\
Male & 30 & 10 & Occipital region \\
Female & 28 & 12 & Parietal and frontal region \\
Male & 26 & 10 & Parietal region \\
Male & 28 & 12 & Parietal and temporal region \\
\hline
\end{tabular}

Table II. Primers used for polymerase chain reaction analysis.

\begin{tabular}{|c|c|c|c|}
\hline Gene name & Primer sequence $\left(5^{\prime} \rightarrow 3^{\prime}\right)$ & Melting temperature $\left({ }^{\circ} \mathrm{C}\right)$ & Product length (bp) \\
\hline \multirow[t]{2}{*}{ TYR } & F: CTCСССТCTTCAGCTGATGT & 57.0 & 150 \\
\hline & R: GCTGCTTTGAGAGGCATCC & & \\
\hline \multirow[t]{2}{*}{ TYRP1 } & F: CCGAAACACAGTGGAAGGTT & 58.9 & 162 \\
\hline & R: TCTGTGAAGGTGTGCAGGAG & & \\
\hline \multirow[t]{2}{*}{ GJB1 } & F: CCTGCACAGACATGAGACCA & 58.9 & 192 \\
\hline & R: CCACCAGCACCATGATTCTG & & \\
\hline \multirow[t]{2}{*}{ PMEL } & F: GATAGGTGCTTTGCTGGCTG & 59.26 & 159 \\
\hline & R: GACACTTGACCACCTCTCCA & & \\
\hline \multirow[t]{2}{*}{ SOX10 } & F: CTGGACCGCACACCTTGG & 59.0 & 197 \\
\hline & R: CTCAGCTCCACCTCCGATAG & & \\
\hline \multirow[t]{2}{*}{ SLC45A2 } & F: CTGCCGACTTCATTGATGGG & 59.0 & 175 \\
\hline & R: TGCAAAGGTAGCGGTAGTGA & & \\
\hline \multirow[t]{2}{*}{ KIT } & F: GGCGGGCATCATGATCAAAA & 59.25 & 160 \\
\hline & R: GCTTGCTTTGGACACAGACA & & \\
\hline \multirow[t]{2}{*}{ TRPM1 } & F: CACCCAGAGCTACCCAACAGA & 59.2 & 165 \\
\hline & R: CGGATATACATGGCTTTATTGGAA & & \\
\hline \multirow[t]{2}{*}{ OCA2 } & F: ACTCTTCTTTGCCCCAGATG & & \\
\hline & R: TCCCAAGACTCTTCAGCAGTG & 59.0 & 158 \\
\hline \multirow[t]{2}{*}{$\beta$-actin } & F: GCGTGACATCAAGGAAGAAGC & 57.5 & 108 \\
\hline & R: CCGTCGGGTAGTTCGTAGCT & & \\
\hline
\end{tabular}

F, forward; R, reverse.

Genes associated with pigmentation and melanin synthesis are predominantly decreased in grey hair follicles. In order to obtain a comprehensive view of genetic changes associated with pigmentation and melanin synthesis, the DEGs identified were classified into pigmentation/melanin-associated GO terms (24). The results indicated that all of the pigmentation/melanin-associated DEGs were decreased in grey hair follicles (Fig. 2A). This suggested that pathways involved in melanin biosynthesis are downregulated in grey hair follicles. These DEGs comprised various genes that are well-known to be responsible for melanin biosynthesis, including TYR, melan-A (MLANA), premelanosome protein (PMEL), TYRP1, SLC45A2, KIT, G protein-coupled receptor 143 (GPR143) and OCA2.

Differentially expressed miRs and TFs regulating pigmentation/melanin-associated DEGs in grey hair follicles. Among the DEGs identified, 13 genes encoded miRs, all of which were significantly increased in grey hair follicles; they accounted for $28 \%$ (13/47) of all upregulated DEGs. It has been previously reported that miRs may be transcribed by RNA polymerase II and have polyA tails (25), and it was therefore likely that the miR-associated RNA-seq reads are from precursors of miRs. Since the majority of the DEGs $(80 / 127 ; 63 \%)$ were decreased in the grey hairs, the changes in the expression of DEGs targeted by these upregulated miR-encoding genes were examined (Fig. 2B). Of note, most of the miR-encoding DEGs (11 out of 13) had the other DEGs as their predicted targets (Table III), the majority of which were significantly decreased in grey compared with black hair follicles. Furthermore, among the miR-targeted DEGs, two genes (syndecan binding protein and KIT) are involved in pigment-associated pathways $(26,27)$.

Another category of regulators affecting gene expression changes are TFs. Therefore, it was then examined whether the DEGs included any TFs and their potential targets. A total of 4 DEGs encoding TFs were identified: AE binding protein 1, 


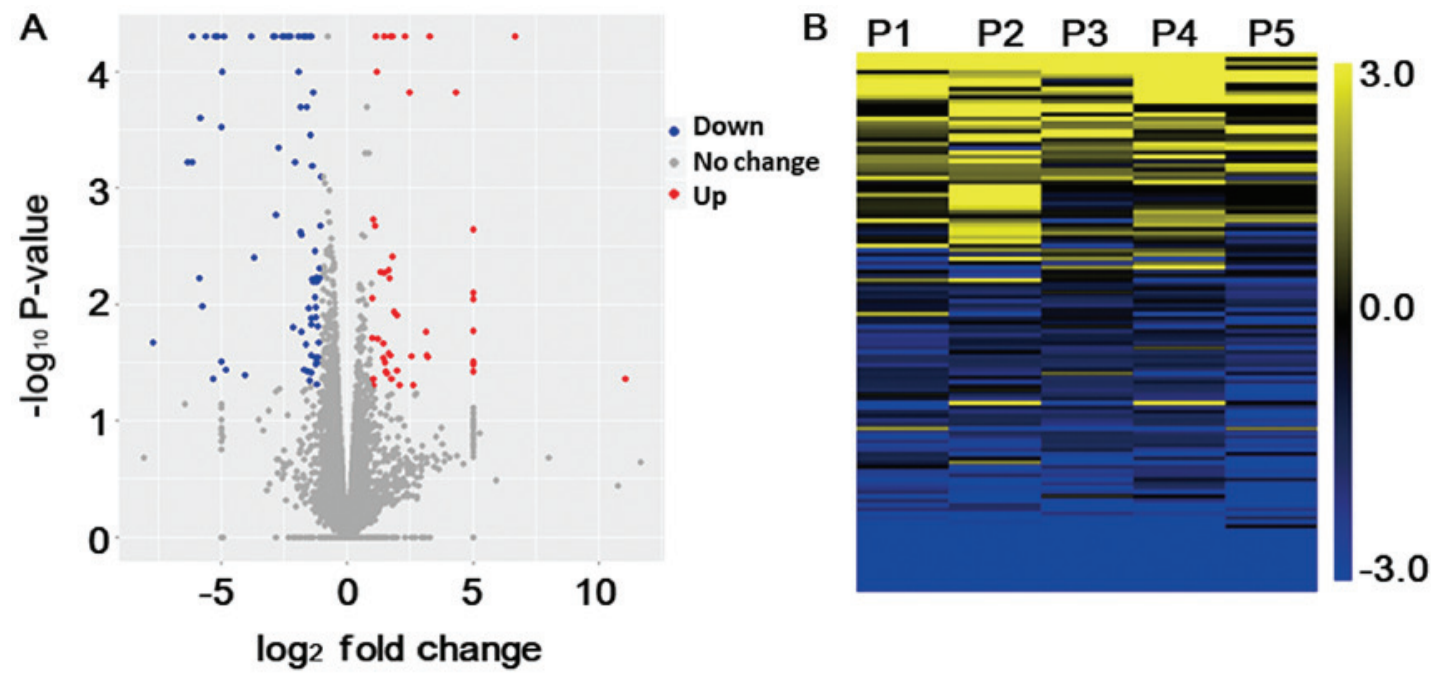

C

Top10 of GO_BP

Secondary metabolite biosynthetic process Pigmentation .

Pigment metabolic process

Pigment biosynthetic process .

Phenol-containing compound metabolic process .

Phenol-containing compound biosynthetic process

Nervous system development

Melanin metabolic process

Melanin biosynthetic process

Developmental pigmentation .

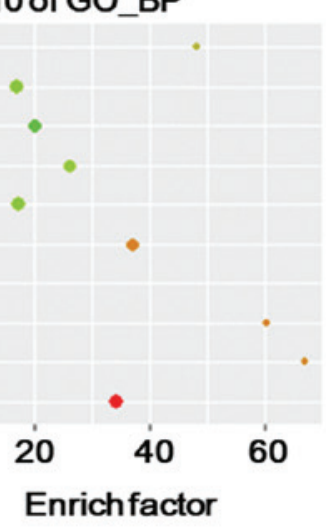

$-\log _{10}$ (Q value)

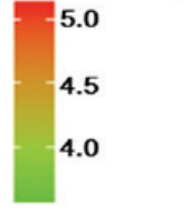

Gene number

- 10

- 15

20

25

D

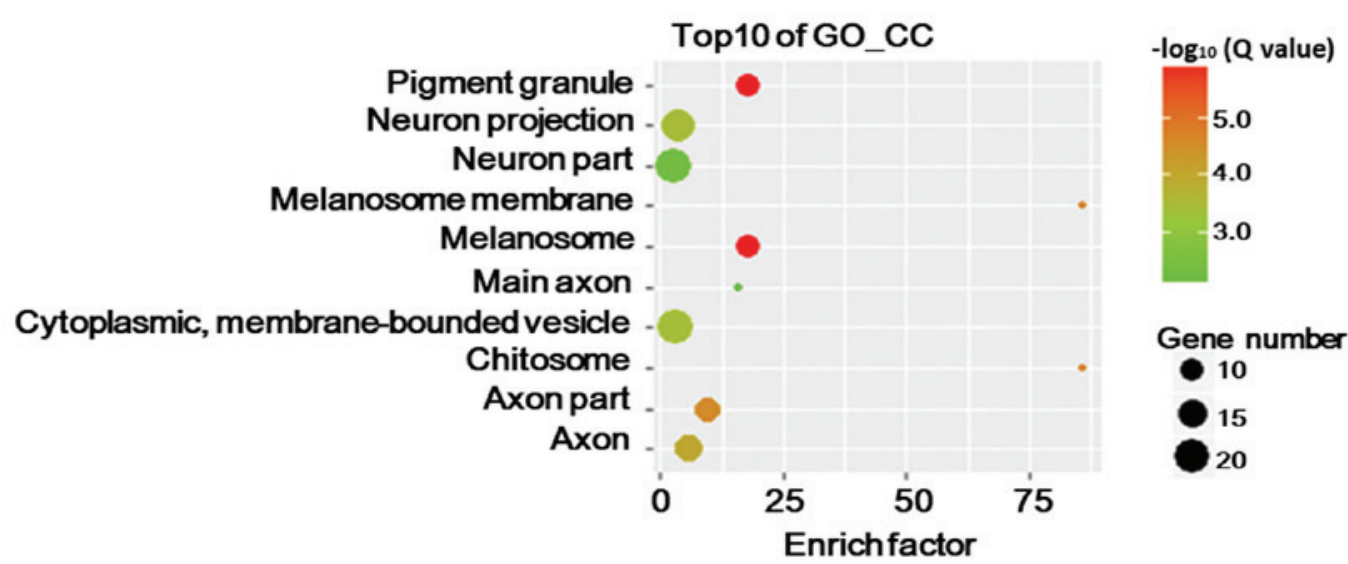

Figure 1. DEGs between black and white hair follicles. (A) Volcano plot of expressed genes (fragments per kilobase per million mapped reads $>1$ ). (B) Heatmap of gene expression changes between black hair and white hair follicles in each subject. Each line represents a gene, each column represents the number per subject, and the colour of the heat map denotes the expression ratio. Functional categories of DEGs between white and black hair follicles. GO analysis was performed to identify the top 10 terms enriched by the DEGs in the categories (C) GO_BP and (D) GO_CC. Circle size represents the number of genes (the larger the circle size, the higher the number of genes). The lowest number of genes in C and D were four and five, respectively. DEG, differentially expressed gene; GO, gene ontology; BP, biological process; CC, cellular component.

coiled coil domain-containing 17, runt-related transcription factor 3 (RUNX3) and SOX10, all of which were significantly decreased in grey hair follicles. Of note, two of these TFs, namely RUNX3 and SOX10, had potential targets among the other DEGs (Table IV), which also tended to be decreased in grey hair follicles (Fig. 2C), indicating an association between the downregulation of the TFs and their potential target genes in grey hair follicles. Transient receptor potential cation channel subfamily M member 1 (TRPM1), which encodes a permeable cation channel that is expressed in melanocytes and has a role in melanin synthesis, was among the downregulated genes regulated by these TFs $(28,29)$. Of note, SOX10 have been proved to have the ability to drive the differentiation of melanocytes (30). 

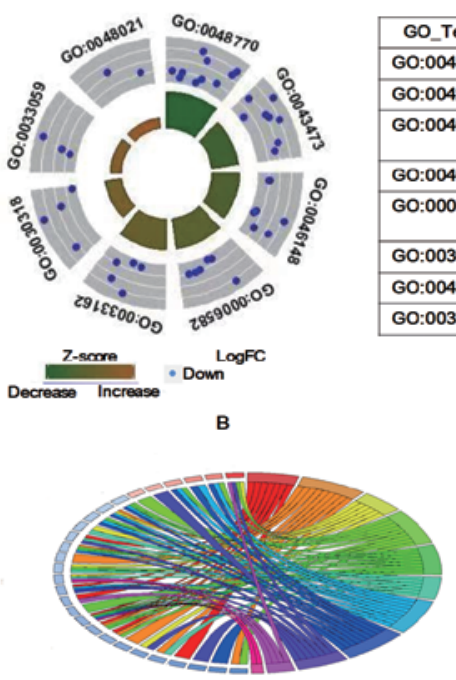

$\frac{\log F C}{-33}$

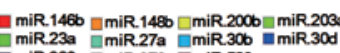

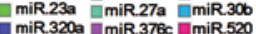

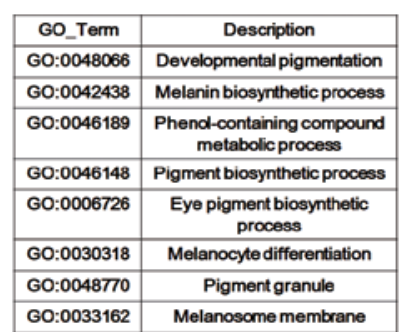

C

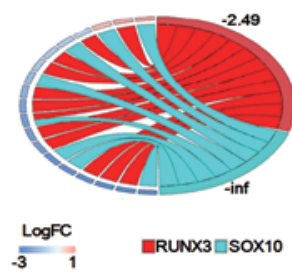

Figure 2. Genes associated with pigmentation and melanin synthesis are predominantly decreased in white hair follicles. (A) GOCircle plot of DEGs associated with pigmentation-associated GO terms. The height of the inner bars indicates the significance of the corresponding terms $(-\log 10$-adjusted $\mathrm{P}$-value), and the color corresponds to the enrichment Z-score (GOplot) The outer ring displays scatterplots of the expression changes $(\log 2 \mathrm{FCl})$ for genes in each term, wherein blue represents decreased expression (B and C) Expression changes of DEGs predicted to be targeted by miRs derived from (B) upregulated miR-encoding genes and (C) downregulated TFs. The gradually changing green-to-pink bars denote the $\log 2 \mathrm{FCl}$ of the target DEGs. The targeted genes are displayed on the left of the circle, while miRs or TFs are presented on the right (red indicates RUNX3 and blue indicates SOX10). miRs or TFs and their predicted targets are linked via ribbons inside. DEG, differentially expressed gene; TF, transcription factor; GO, gene ontology; FC, fold change; miR, microRNA; SOX, sex-determining region Y box; RUNX, runt-related TF.

PPI network centers for genes associated with melanin synthesis. A protein usually interacts with other proteins to function properly. To obtain a systematic view of the biological roles of the DEGs in grey hair follicles, the PPI networks were analyzed using DEGs by means of the STRING database. The result indicated that DEGs were involved in a PPI network centering on genes associated with melanin biosynthesis (Fig. 3), including TYR, TYRP1, MLANA, GPR143, OCA2, PMEL, SLC24A5 and SLC45A2. Further genes that are not involved in melanin synthesis, including KIT ligand, checkpoint kinase 1 and integrin subunit $\beta 3$, were also identified. However, they may affect the melanin biosynthesis process and ultimately affect hair pigmentation through interacting with genes directly involved in melanin biosynthesis.

Validation of RNA-seq data by $R T-q P C R$. In order to verify gene expression differences determined via RNA-seq analysis, 9 randomly selected genes [TYR, PMEL, TYRP1, gap junction $\beta 1$ (GJB1), SOX10, SLC45A2, KIT, TRPM1 and OCA2] were detected by RT-qPCR. As presented in Fig. 4, the abscissa represents different samples, and ordinates are the relative expression value of grey vs. black hair. The results indicated that the expressional changes of all of these genes were consistent with those detected by RNA-seq analysis, thus confirming that all genes were consistently downregulated in grey vs. black hair follicles.

\section{Discussion}

Hair graying, particularly premature hair graying, changes the appearance of affected individuals in a mostly undesired manner and has attracted the attention of researchers. To date, the underlying causes have remained largely elusive. In the present study, a genome-wide RNA-seq profiling analysis was performed using grey and black hair follicles from the same individuals with premature hair graying. It was revealed that pigment synthesis pathways were significantly impaired in grey vs. black hair, with a significantly decreased expression of multiple key genes crucial for the stability, trafficking and proliferation of melanocytes (31). The present results support the theory that premature graying may occur due to exhaustion of the melanocytes' capability to produce hair pigmentation.

Previous studies have suggested that premature hair graying is associated with factors affecting melanogenesis, including nutritional deficiencies (32), insufficient neuroendocrine stimulation (33) and ionic signaling across melanosomes (34). The identified DEGs included those with similar functions with this regard, e.g. MCHR1, TRPM1 and SLC45A2. MCHR1 acts as a receptor for melanin-concentrating hormone (35), TRPM1 regulates pigmentation at the plasma membrane level (29) and SLC45A2 modulates the melanosomal $\mathrm{pH}$ for optimal TYR activity required for melanogenesis (36). Of note, the present study identified that GJB1 was downregulated in grey vs. black hair follicles, which known to contribute to pigment transfer between melanocytes and neighboring keratinocytes (37).

While pigmentation-associated pathways are impaired in grey hair follicles, the underlying mechanisms of their inhibition/damage during hair greying remain to be elucidated. Of note, all of the 13 DEGs encoding miRs identified in the present study were significantly upregulated in grey hair follicles, indicating a substantial increase in the abundance of the corresponding mature miRs. Furthermore, the majority of the DEGs predicted to be targets of these miRs were significantly decreased in grey hair follicles, which is in line with the known mechanism that miRs reduce the expression of their target genes.

Another reason could be the decreased TFs in grey hair follicles. Among the four decreased TFs expressed in gray hair, two TFs (RUNX3 and SOX10) had potential target genes in other DEGs, which were also downregulated in gray hair, indicating that the downregulation of TFs were associated with their potential target genes in gray hair follicles. In addition, GJB1 was co-expressed with a crucial node, SOX10, in the PPI network. It has been reported that SOX10, in synergy with early growth response 2, may activate GJB1 in melanocytes, which may cause and alteration in melanogenesis $(38,39)$.

Another point worth mentioning is that a relatively high fraction of DEGs was associated with the nervous system, including potassium voltage-gated channel subfamily $Q$ member 2, basic helix-loop-helix family member e41, prostaglandin D2 synthase and centrosomal protein of 152 $\mathrm{kDa}$, which are thought to be involved in controlling the circadian rhythm. Defects in these genes have been reported to be associated with a short sleep phenotype $(40,41)$. It is widely accepted that nerve signaling defects, including a disturbed sleeping ability, may lead to hair graying, which 
Table III. Differentially expressed genes that are predicted targets of miRs in Table IV.

\begin{tabular}{llcccr}
\hline Gene ID & Gene & FPKM in black hair & FPKM in white hair & Log2|FCl & P-value \\
\hline ENSG00000157404 & KIT & 4.22 & 0.14 & -4.88 & 0.00005 \\
ENSG00000136040 & PLXNC1 & 6.36 & 0.46 & -3.79 & 0.00005 \\
ENSG00000104177 & MYEF2 & 1.29 & 0.17 & -2.92 & 0.00005 \\
ENSG00000020633 & RUNX3 & 1.48 & 0.26 & -2.49 & 0.00005 \\
ENSG00000145335 & SNCA & 6.53 & 1.28 & -2.35 & 0.00005 \\
ENSG00000071575 & TRIB2 & 2.72 & 0.56 & -2.28 & 0.00005 \\
ENSG00000123095 & BHLHE41 & 5.33 & 1.2 & -2.16 & 0.01575 \\
ENSG00000130558 & OLFM1 & 1.27 & 0.33 & -1.94 & 0.00010 \\
ENSG00000137575 & SDCBP & 10.59 & 2.79 & -1.93 & 0.00005 \\
ENSG00000048740 & CELF2 & 1.84 & 0.49 & -1.91 & 0.00005 \\
ENSG00000197283 & SYNGAP1 & 20.4 & 5.71 & -1.84 & 0.00240 \\
ENSG00000166173 & LARP6 & 1.39 & 0.4 & -1.8 & 0.01725 \\
ENSG00000026025 & VIM & 26.35 & 7.8 & -1.76 & 0.00005 \\
ENSG00000204764 & RANBP17 & 1.35 & 0.43 & -1.65 & 0.02195 \\
ENSG00000115825 & PRKD3 & 6.13 & 2.31 & -1.41 & 0.01485 \\
ENSG00000082397 & EPB41L3 & 1.85 & 0.73 & -1.34 & 0.00015 \\
ENSG00000173482 & PTPRM & 2.16 & 0.88 & -1.29 & 0.00870 \\
ENSG00000153823 & PID1 & 1.42 & 0.59 & -1.26 & 0.00345 \\
ENSG00000115414 & FN1 & 1.68 & 0.72 & -1.23 & 0.01050 \\
ENSG00000185008 & ROBO2 & 1.5 & 0.65 & -1.2 & 0.04845 \\
ENSG00000086289 & EPDR1 & 3.04 & 1.42 & -1.09 & 0.00595 \\
ENSG00000116641 & DOCK7 & 11.49 & 5.51 & -1.06 & 0.00210 \\
ENSG00000249859 & PVT1 & 10.11 & 20.29 & 1 & 0.01935 \\
ENSG00000135346 & CGA & 14.88 & 32.89 & 1.14 & 0.00005 \\
ENSG00000137941 & TTLL7 & 1.77 & 1.44 & 1.32 & 0.00525 \\
ENSG00000105784 & RUNDC3B & 0.44 & 1.21 & 1.7 & 0.00595 \\
ENSG00000113532 & ST8SIA4 & 0.33 & 1.84 & 1.88 & 0.01150 \\
ENSG00000151967 & SCHIP1 & 0.09 & 4.32 & 0.00015 \\
\hline
\end{tabular}

miR, microRNA; FPKM, fragments per kilobase per million mapped reads; FC, fold change.

Table IV. Differentially expressed genes targeted by differential expression of genes encoding transcription factors (RUNX3 and sex-determining region Y box 10).

\begin{tabular}{llccrr}
\hline Gene ID & Gene & FPKM in black hair & FPKM in white hair & Log2|FCl & P-value \\
\hline ENSG00000020633 & RUNX3 & 1.48 & 0.26 & -2.49 & 0.00005 \\
ENSG00000048740 & CELF2 & 1.84 & 0.49 & -1.91 & 0.00005 \\
ENSG00000069424 & KCNAB2 & 4.88 & 0.99 & -2.31 & 0.00005 \\
ENSG00000086289 & EPDR1 & 3.04 & 1.42 & -1.09 & 0.00595 \\
ENSG00000089101 & CFAP61 & 1.42 & 0.25 & -2.52 & 0.00005 \\
ENSG00000091986 & CCDC80 & 4.3 & 8.75 & 1.03 & 0.00185 \\
ENSG00000104177 & MYEF2 & 1.29 & 0.17 & -2.92 & 0.00005 \\
ENSG00000115414 & FN1 & 1.68 & 0.72 & -1.23 & 0.01050 \\
ENSG00000123560 & PLP1 & 3.13 & 0.06 & -5.74 & 0.01035 \\
ENSG00000130751 & NPAS1 & 0.55 & 1.47 & 1.42 & 0.02150 \\
ENSG00000134160 & TRPM1 & 8.52 & 0.15 & -5.83 & 0.00025 \\
ENSG00000136732 & GYPC & 1.43 & 1.49 & -4.05 & 0.04075 \\
ENSG00000154277 & UCHL1 & 8.49 & 0.88 & -2.52 & 0.00005 \\
ENSG00000173482 & PTPRM & 2.16 & 16.75 & -1.29 & 0.00870 \\
ENSG00000184724 & KRTAP6-1 & 6.05 & 0.53 & -1.47 & 0.00005 \\
ENSG00000187045 & TMPRSS6 & 1.19 & 0.37 & -2.85 & 0.02810 \\
ENSG00000197415 & VEPH1 & 2.63 & 0.43 & -1.65 & 0.00170 \\
ENSG00000204764 & RANBP17 & 1.35 & 0.02 & -6.14 & 0.00060 \\
ENSG00000221887 & HMSD & 1.58 & & &
\end{tabular}

FPKM, fragments per kilobase per million mapped reads; RUNX, runt-related transcription factor; FC, fold change. 


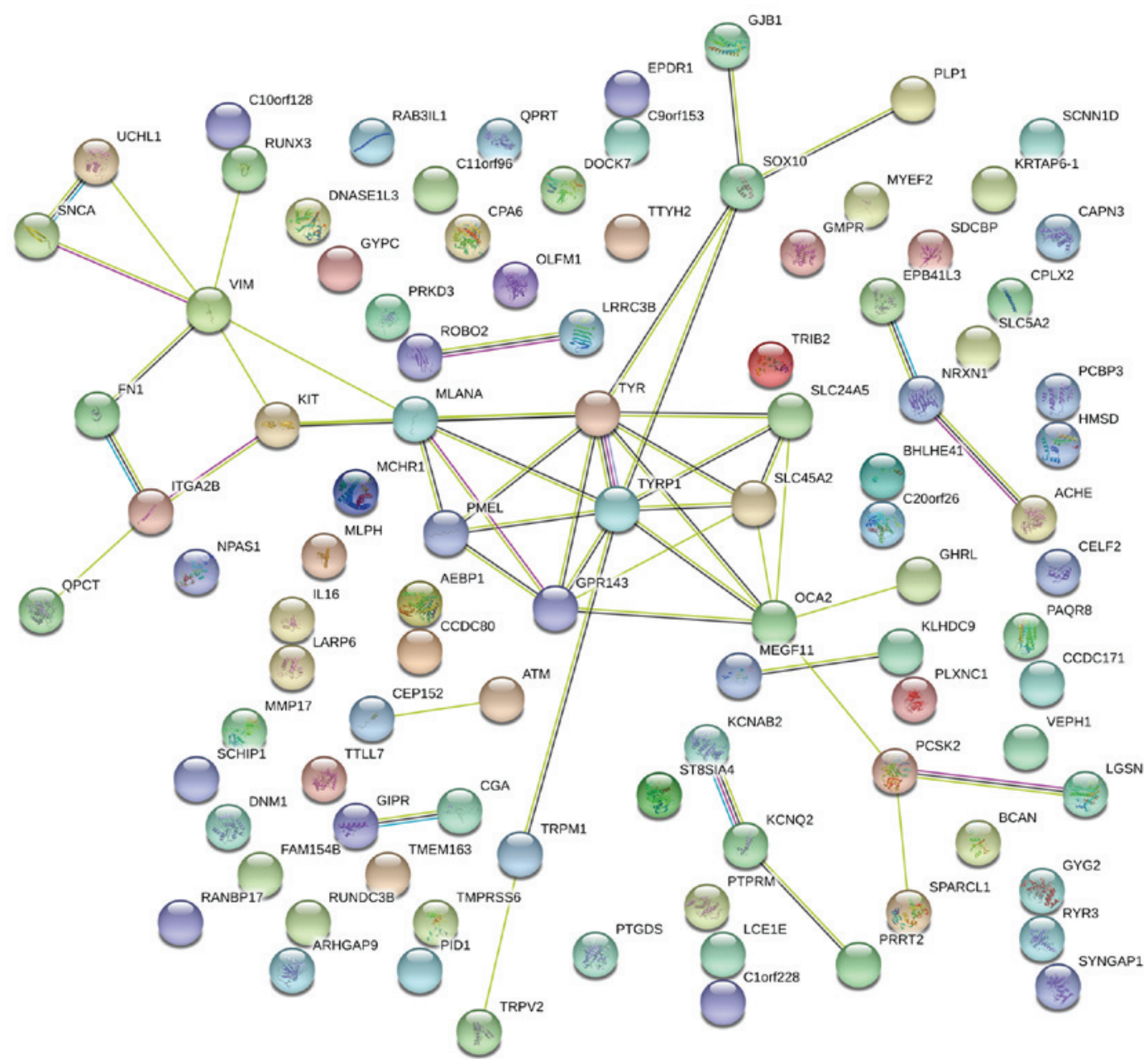

Figure 3. Protein-protein interaction network of the DEGs (TYR, TYRP1, MLANA, GPR143, OCA2, PMEL, SLC24A5 and SLC45A2). The network was generated using the STRING database by inputting the gene names of the DEGs between white hair and black hair follicles. DEG, differentially expressed gene.
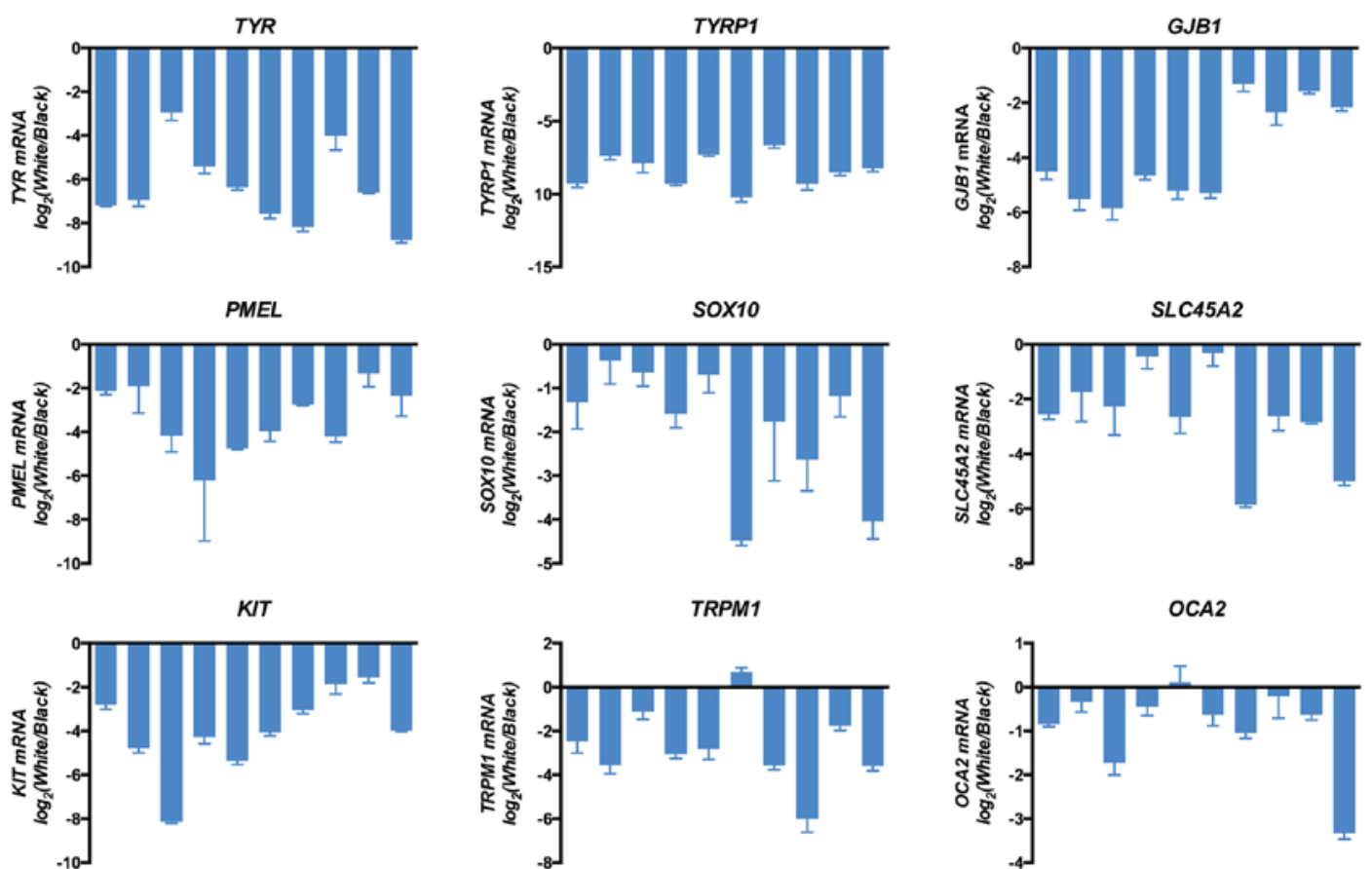

Figure 4. Validation of 9 differentially expressed genes in white vs. black hair follicles. The abundance of target genes was normalized relative to the abundance of the $\beta$-actin gene. The values were calculated by using the $2^{-\Delta \Delta C q}$ method. RNA was extracted from the gray and black hair of 10 patients. Each list indicates the $\log$ ratio of gray/black hair gene expression in each patient. A total of 10 columns present the log ratio. Nine genes are selected for quantitative analysis. 
probably functions through its further effects on the nervous system and downstream pigmentation pathways (42). While it remains elusive whether and how the nervous system contributes to premature hair graying, the present results may indicate a novel aspect regarding the causes of hair graying. Since hair graying has been considered to be associated with aging of the hair follicle pigmentation system (43), the JenAge Ageing Factor Database (44) was searched for the DEGs in grey hair identified in the present study. A total of 5 genes were included in the online database, namely acetylcholine esterase, ATM, proprotein convertase subtilisin/kexin type 2, ubiquitin C-terminal hydrolase L1 and ventricular zone expressed PH domain containing 1, all of which had a decreased expression in white hair follicles, implying the decline of melanocyte-associated processes.

In conclusion, the present study was the first, to the best of our knowledge, to perform a genome-wide transcriptome profiling of human hair follicles affected by premature hair graying, which uncovered that damage of the melanin biosynthesis pathway was the direct cause of the decline hair pigmentation and the resulting hair graying. Furthermore, it was indicated that deregulated miRs and TFs, as well as neural disturbances, may be underlying causes. The present study provided multiple clues worthy of subsequent study to elucidate the mechanisms underlying canities and human premature hair graying. However, the present study is limited as only the processes occurring in subjects with premature hair greying were assessed, while the genetic predisposition to hair greying was not be determined. The genetic differences between subjects with premature hair greying and normal individuals should be assessed to identify the genes that are the primary cause of this condition.

\section{Acknowledgements}

The authors would like to thank Dr Shiming Wang, Dr Cong Huai and Dr Hexige Saiyin (School of Life Sciences, Fudan University, Shandhai, China), as well as Dr Xiaotian Wang (Thermo Fisher Scientific, Inc.) for their assistance in sample collection and RNA-seq library construction.

\section{Funding}

The present study was supported by the National Natural Science Foundation (grant no. 31571371) and the National Key Research and Development Plan (grant no. 2017YFC090750).

\section{Availability of data and materials}

The data used and/or analyzed during the present study are available from the corresponding author on reasonable request.

\section{Authors' contributions}

DL, TN and HC conceived and designed the experiments. YB, XS and LY collected hair follicles, prepared RNA samples and constructed RNA-Seq libraries. GW analyzed transcriptome data. YB and LY performed qRT-PCR. YB and GW wrote the paper. All authors read and approved the final version of the manuscript.

\section{Ethical approval and consent to participate}

The present study was performed according to the declaration of Helsinki and was approved by the Research Ethics Committee at Fudan University (Shanghai, China). Informed consent was obtained from each participant prior to enrollment.

\section{Patient consent for publication}

Not applicable.

\section{Competing interests}

The authors declare that they have no competing interests.

\section{References}

1. Panhard S, Lozano I and Loussouarn G: Greying of the human hair: A worldwide survey, revisiting the '50' rule of thumb. $\mathrm{Br}$ J Dermatol 167: 865-873, 2012.

2. Tobin DJ: Human hair pigmentation-biological aspects. Int J Cosmet Sci 30: 233-257, 2008.

3. Tobin DJ and Paus R: Graying: Gerontobiology of the hair follicle pigmentary unit. Exp Gerontol 36: 29-54, 2001.

4. Pandhi D and Khanna D: Premature graying of hair. Indian J Dermatol Venereol Leprol 79: 641-653, 2013.

5. Slominski A, Wortsman J, Plonka PM, Schallreuter KU, Paus R and Tobin DJ: Hair follicle pigmentation. J Invest Dermatol 124: 13-21, 2005.

6. Slominski A, Paus R and Costantino R: Differential expression and activity of melanogenesis-related proteins during induced hair growth in mice. J Invest Dermatol 96: 172-179, 1991.

7. Slominski A, Paus R, Plonka P, Chakraborty A, Maurer M, Pruski D and Lukiewicz S: Melanogenesis during the anagen-catagen-telogen transformation of the murine hair cycle. J Invest Dermatol 102: 862-869, 1994.

8. Tobin DJ: The cell biology of human hair follicle pigmentation. Pigment Cell Melanoma Res 24: 75-88, 2011.

9. Rees JL: Genetics of hair and skin color. Annu Rev Genet 37: 67-90, 2003.

10. Sturm RA: Molecular genetics of human pigmentation diversity. Hum Mol Genet 18: R9-R17, 2009.

11. Sabeti PC, Varilly P, Fry B, Lohmueller J, Hostetter E, Cotsapas C, Xie X, Byrne EH, McCarroll SA, Gaudet R, et al: Genome-wide detection and characterization of positive selection in human populations. Nature 449: 913-918, 2007.

12. Han J, Kraft P, Nan H, Guo Q, Chen C, Qureshi A, Hankinson SE, Hu FB, Duffy DL, Zhao ZZ, et al: A genome-wide association study identifies novel alleles associated with hair color and skin pigmentation. PLoS Genet 4: e1000074, 2008.

13. Sulem P, Gudbjartsson DF, Stacey SN, Helgason A, Rafnar T, Jakobsdottir M, Steinberg S, Gudjonsson SA, Palsson A, Thorleifsson G, et al: Two newly identified genetic determinants of pigmentation in Europeans. Nat Genet 40: 835-837, 2008.

14. Zhao Z, Duffy D, Thomas S, Martin NG, Hayward NK and Montgomery GW: Polymorphisms in the syntaxin 17 gene are not associated with human cutaneous malignant melanoma. Melanoma Res 19: 80-85, 2009.

15. Diaz de Leon A, Cronkhite JT, Yilmaz C, Brewington C, Wang R, Xing C, Hsia CCW and Garcia CK: Subclinical lung disease, macrocytosis, and premature graying in kindreds with telomerase (TERT) mutations. Chest 140: 753-763, 2011.

16. Kwon MJ, Lee KY, Lee HW, Kim JH and Kim TY: SOD3 variant, R213G, altered SOD3 function, leading to ROS-mediated inflammation and damage in multiple organs of premature aging mice. Antioxid Redox Signal 23: 985-999, 2015.

17. Lin BD, Mbarek H, Willemsen G, Dolan CV, Fedko IO, Abdellaoui A, de Geus EJ, Boomsma DI and Hottenga JJ: Heritability and genome-wide association studies for hair color in a dutch twin family based sample. Genes (Basel) 6: 559-576, 2015.

18. Dobin A, Davis CA, Schlesinger F, Drenkow J, Zaleski C, Jha S, Batut P, Chaisson M and Gingeras TR: STAR: Ultrafast universal RNA-seq aligner. Bioinformatics 29: 15-21, 2013. 
19. Trapnell C, Roberts A, Goff L, Pertea G, Kim D, Kelley DR, Pimentel H, Salzberg SL, Rinn JL and Pachter L: Differential gene and transcript expression analysis of RNA-seq experiments with TopHat and Cufflinks. Nat Protoc 7: 562-578, 2012.

20. Benjamini Y and Hochberg Y: Controlling the false discovery rate-a practical and powerful approach to multiple testing. J Royal Stat Soc Series B-Methodol 57: 289-300, 1995.

21. Li JH, Liu S, Zhou H, Qu LH and Yang JH: starBase v2.0 Decoding miRNA-ceRNA, miRNA-ncRNA and protein-RNA interaction networks from large-scale CLIP-Seq data. Nucleic Acids Res 42 (Database issue): D92-D97, 2014.

22. Balwierz PJ, Pachkov M, Arnold P, Gruber AJ, Zavolan M and van Nimwegen E: ISMARA: Automated modeling of genomic signals as a democracy of regulatory motifs. Genome Res 24 869-884, 2014

23. Szklarczyk D, Franceschini A, Kuhn M, Simonovic M, Roth A, Minguez P, Doerks T, Stark M, Muller J, Bork P, et al: The STRING database in 2011: Functional interaction networks of proteins, globally integrated and scored. Nucleic Acids Res 39 (Database issue): D561-D568, 2011.

24. Walter W, Sánchez-Cabo F and Ricote M: GOplot: An R package for visually combining expression data with functional analysis. Bioinformatics 31: 2912-2914, 2015.

25. Lee Y, Kim M, Han J, Yeom KH, Lee S, Baek SH and Kim VN: MicroRNA genes are transcribed by RNA polymerase II. EMBO J 23: 4051-4060, 2004.

26. Aissaoui H, Prévost C, Boucharaba A, Sanhadji K, Bordet JC, Négrier C and Boukerche H: MDA-9/syntenin is essential for factor VIIa-induced signaling, migration, and metastasis in melanoma cells. J Biol Chem 290: 3333-3348, 2015.

27. Jiang S, Yu X and Dong C: MiR-137 affects melanin synthesis in mouse melanocyte by repressing the expression of c-Kit and Tyrp2 in SCF/c-Kit signaling pathway. Biosci Biotechnol Biochem 80: 2115-2121, 2016.

28. Gees M, Owsianik G, Nilius B and Voets T: TRP channels Compr Physiol 2: 563-608, 2012

29. Oancea E, Vriens J, Brauchi S, Jun J, Splawski I and Clapham DE: TRPM1 forms ion channels associated with melanin content in melanocytes. Sci Signal 2: ra21, 2009.

30. Harris ML, Buac K, Shakhova O, Hakami RM, Wegner M, Sommer L and Pavan WJ: A dual role for SOX10 in the maintenance of the postnatal melanocyte lineage and the differentiation of melanocyte stem cell progenitors. PLoS Genet 9: e1003644, 2013.

31. D'Mello SA, Finlay GJ, Baguley BC and Askarian-Amiri ME: Signaling pathways in melanogenesis. Int J Mol Sci 17: E1144, 2016

32. Fatemi Naieni F, Ebrahimi B, Vakilian HR and Shahmoradi Z: Serum iron, zinc, and copper concentration in premature graying of hair. Biol Trace Elem Res 146: 30-34, 2012.
33. Paus R: A neuroendocrinological perspective on human hair follicle pigmentation. Pigment Cell Melanoma Res 24: 89-106, 2011.

34. Bellono NW and Oancea EV: Ion transport in pigmentation. Arch Biochem Biophys 563: 35-41, 2014.

35. Kemp EH, Waterman EA, Hawes BE, O'Neill K, Gottumukkala RV, Gawkrodger DJ, Weetman AP and Watson PF: The melanin-concentrating hormone receptor 1, a novel target of autoantibody responses in vitiligo. J Clin Invest 109: 923-930, 2002.

36. Dooley CM, Schwarz H, Mueller KP, Mongera A, Konantz M, Neuhauss SC, Nüsslein-Volhard C and Geisler R: Slc45a2 and $\mathrm{V}$-ATPase are regulators of melanosomal $\mathrm{pH}$ homeostasis in zebrafish, providing a mechanism for human pigment evolution and disease. Pigment Cell Melanoma Res 26: 205-217, 2013.

37. Joshi PG, Nair N, Begum G, Joshi NB, Sinkar VP and Vora S: Melanocyte-keratinocyte interaction induces calcium signalling and melanin transfer to keratinocytes. Pigment Cell Res 20: 380-384, 2007.

38. LeBlanc SE, Ward RM and Svaren J: Neuropathy-associated Egr2 mutants disrupt cooperative activation of myelin protein zero by Egr2 and Sox10. Mol Cell Biol 27: 3521-3529, 2007.

39. Ludwig A, Rehberg S and Wegner M: Melanocyte-specific expression of dopachrome tautomerase is dependent on synergistic gene activation by the Sox 10 and Mitf transcription factors. FEBS Letters 556: 236-244, 2004.

40. Lee IC, Yang JJ and Li SY: A KCNQ2 E515D mutation associated with benign familial neonatal seizures and continuous spike and waves during slow-wave sleep syndrome in Taiwan. J Formos Med Assoc 116: 711-719, 2017.

41. Pellegrino R, Kavakli IH, Goel N, Cardinale CJ, Dinges DF, Kuna ST, Maislin G, Van Dongen HP, Tufik S, Hogenesch JB, et al: A novel BHLHE41 variant is associated with short sleep and resistance to sleep deprivation in humans. Sleep 37: 1327-1336, 2014.

42. Anderson NE and Haas LF: Neurological complications of Werner's syndrome. J Neurol 250: 1174-1178, 2003.

43. Tobin DJ: Aging of the hair follicle pigmentation system. Int J Trichology 1: 83-93, 2009.

44. Huhne R, Thalheim T and Sühnel J: AgeFactDB-the JenAge ageing factor database-towards data integration in ageing research. Nucleic Acids Research (Database issue) 42: D892-D896, 2014. 